\title{
INFLUENCIAS DEL LINEAMIENTO TRANSTENSIVO SANTA LUCÍA-AIGUÁ-MERÍN SOBRE EL EMPLAZAMIENTO DEL MAGMATISMO ALCALINO CRETÁCICO DEL CRATÓN LA PLATA (URUGUAY): APORTES A LA PROSPECCIÓN DIAMANTÍFERA
}

\author{
EDUARDO A. ROSSELLO ${ }^{1}$, GERARDO VEROSLAVSKY ${ }^{2}$ \& HÉCTOR DE SANTA ANA ${ }^{2,3}$
}

\begin{abstract}
The Santa Lucía-Aigua-Merín Lineament (SaLAM) is a $450 \mathrm{~km}$ long structural transtensive corridor running across the La Plata Craton in southeastern Uruguay (flanked by the shores of the Río de la Plata, near Montevideo, and Laguna Merín, near Brazil). This first order structure controls; i) two pull-apart depocenters (Santa Lucía and Laguna Merín basins) at their extremes, and ii) the emplacement of Mesozoic remains and alkaline intrusions represented by a thick crystalline basement block, in the intermediate segment. The SaLAM exhibits two main tectonic phases: i) a Jurassic to Early Cretaceous extensional phase, represented by intraplate magmatism, and ii) an Aptian-Albian dextral transtensional phase. The central segment of the SaLAM Lineament determines an interesting tectonic scenario to prospect kimberlite bearing rocks in the thick crystalline basement of the Uruguay.
\end{abstract}

Keywords: Santa Lucía-Aiguá-Merín Lineament, Mesozoic, transtensional tectonics, alkaline magmatism emplacement, La Plata Craton, Uruguay.

INTRODUCCIÓN El Lineamiento Santa Lucía-AiguáMerín (SaLAM) constituye un corredor tectónico transtensivo que afectó al basamento cristalino del Cratón La Plata del Uruguay (Dalla Salda et al. 1988). Se considera al SaLAM como la expresión geotectónica de una zona extensiva cortical con una llamativa disposición subparalela al desarrollo temprano de la apertura Atlántica.

Su funcionamiento distensivo sobre una corteza continental anormalmente engrosada a facilitado el rápido ascenso y emplazamiento de magmatismo alcalino que tiene potencial para alumbrar rocas kimberlíticas que puedan asociarse a las aún esporádicas menciones informales de hallazgos diamantíferos detríticos en el territorio uruguayo.

El Lineamiento SaLAM El SaLAM exhibe un diseño mayormente rectilíneo de unos $450 \mathrm{~km}$ de longitud, de rumbo general $\mathrm{N} 70^{\circ}$, que se sobreimpone a la estructuración precámbrica heterogénea del basamento cristalino donde se individualizan tres grandes terrenos o unidades geotectónicas de edad precámbrica dispuestos submeridianalmente: i) Piedra Alta, al Oeste, ii) Nico Pérez, al Centro y iii) Dom Feliciano o Cuchilla Dionisio, al Este (véanse detalles regionales en Bossi et al. 1998).

El funcionamiento extensional desde el Jurásico y transcurrente dextral durante el Cretácico tardío (Rossello et al. 2001) controló en pandeos derechos dilatantes de sus extremos la formación de las cuencas Santa Lucía (hacia el sudoeste) y Laguna Merín (hacia el noreste), tal como lo esquematiza la Figura 1. En su porción central, se asocia temporo-espacialmente con el emplazamiento y preservación de los afloramientos volcánicos e intrusivos alkalinos y sedimentarios mesozoicos que se desarrollan próximos o en las fosas

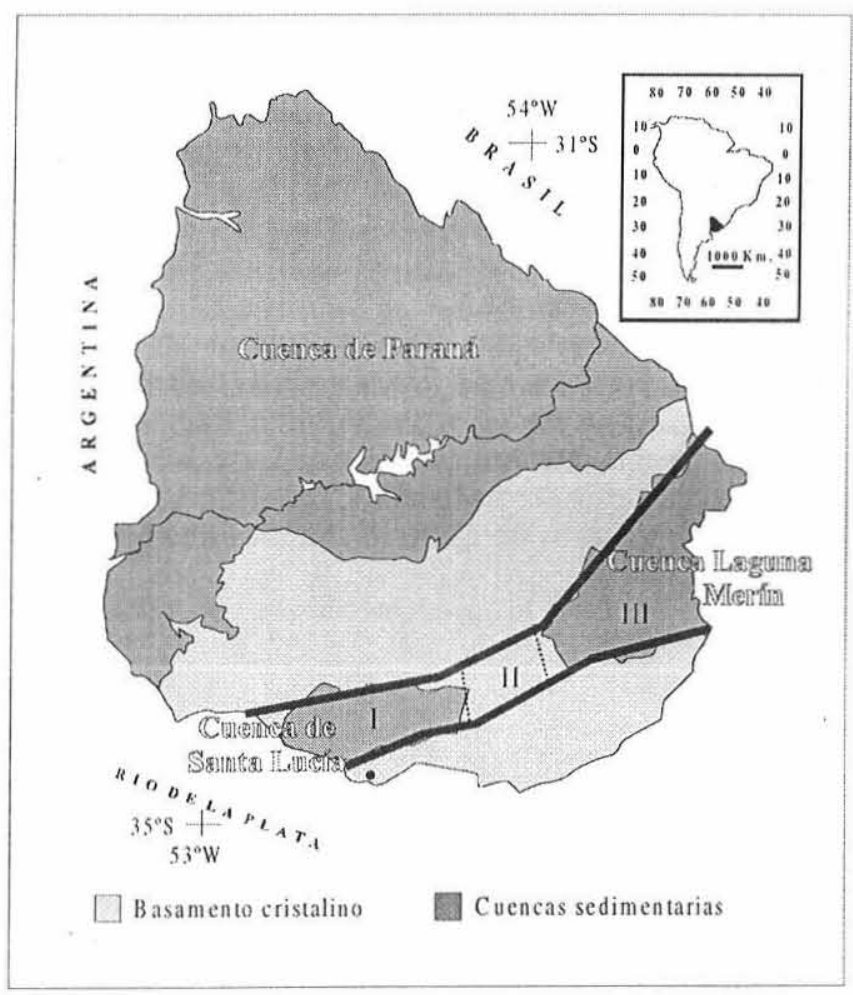

Figura I - Localización relativa del Lineamiento Santa LucíaAigua-Merín sobre la porción sudoriental de la República Oriental del Uruguay. I: Sector Sudoccidental (Cuenca Santa Lucía), II, Sector Intermedio, III: Sector Nororiental (Cuenca Laguna Merín). Tomado de Rossello et al. (2001).

I CONICET - Departamento de Ciencias Geológicas, Universidad de Buenos Aires, Pabellón II, Ciudad Universitaria, 1428 - Buenos Aires - Argentina. E-mail: rossello@gl.fcen.uba.ar

2 Departamento Geología (INGEPA), Facultad de Ciencias, Universidad de la República, Iguá 4225. CP 11400. Mnontevideo, Uruguay. E-mail: gerardo@fcien.edu.uy

3 ANCAP División Investigación y Desarrollo. Av. Lib. Gral. Lavalleja y Paysandú. CP 1090. Montevideo, Uruguay. hdsa@fcien.edu.uy : 
tectónicas de Minas, Aiguá, Lascano y Velázquez.

El segmento central del SaLAM afecta al basamento cristalino del Cratón La Plata complejamente estructurado y engrosado desde el Arqueano donde Introcaso \& Huerta (1982), estiman a partir de datos gravimétricos del Servicio Geográfico Militar (1973) una litósfera con 110 km de espesor que contrasta con lo observado en el extremo nororiental que registra la anomalía gravimétrica positiva de Bouguer más importante del territorio continental del Uruguay que llega a valores superiores a los $60 \mathrm{mGal}$.

En la evolución del SaLAM, se pueden reconocer dos fases tectónicas (Rossello et al. 2001): i) una inicial Juro-eocretácica, claramente distensiva; y ii) otra posterior, a partir de AptianoAlbiano, donde se establece el carácter transcurrente dextral. El abandono de la extensión más pura del SaLAM debe haberse generado, de modo transicional, por una mayor eficiencia mecánica de las antiguas discontinuidades submeridianales localizadas hacia el oriente del terreno Dom Feliciano que pudieron haber canalizado la apertura y creación de la corteza oceánica Atlántica.

El carácter transcurrente dextral del SaLAM está determinado (Rossello et al. 2001), a escala regional, por el arreglo espacial escalonado de diferentes rasgos extensivos dispuestos con rumbos $\mathrm{N} 100^{\circ}$ a $130^{\circ}$ a lo largo del corredor $\mathrm{N} 70^{\circ}$ : i) los depocentros y geometría de sedimentitas y de efusiones volcánicas contemporáneas, ii) el arrumbamiento de los ejes longitudinales sublatitudinales a ESE-ONO de las fosas estrechas limitadas por fallas normales y con relleno volcanosedimentario de Minas, Aiguá, Colón, Lascano; iii) la deformación de los filones fluoríticos y cuarzosos, en el margen austral de la fosa Aiguá; iv) y la intensa fracturación distensiva NO del complejo largo de toda su extensión.

El funcionamiento del SaLAM como una transcurrencia dextral estaría relacionado a la actuación de esfuerzos de alivio de intraplaca vinculados al inicio de la fragmentación del Gondwana y a la fase de separación de la placa Sudamericana y Africana a partir del Aptiano. Al respecto, varios modelos señalan los procesos de apertura atlántica que proponen un régimen de esfuerzos que supone la actuación de zonas con transcurrencias dextrales que se desarrollan sobre discontinuidades precámbricas.

Sobre la traza central del SaLAM, donde la corteza continental es muy potente se reconoce la presencia de magmatismo alcalino cretácico en forma de stocks, diques y coladas (Gómez-Rifas \& Masquelin-Arcelus 1996, Muzio et al. 1999; Pirelli 1999), que puede regionalmente asociarse al magmatismo relacionado con la apertura Atlántica (Turner et al. 1994; Peate 1997). Sin embargo, hacia su porción nororiental de la Cuenca Laguna Merín, la actividad magmática fue más intensa ya que al menos $1.000 \mathrm{~m}$ de basaltos fueron registrados indicando una transición hacia un ambiente de placa océanica. Esta fuerte variación podría justificarse a partir de la necesidad de compensar isostáticamente los adelgazamientos corticales marginales de la placa sudamericana.

CONCLUSIONES A partir de estudios gravimétricos y tectónicos regionales puede identificarse la presencia de un basamento cristalino pre-Mesozoico que integra una corteza continental substancialmente gruesa en gran parte del territorio central del Uruguay. Por otro lado, la presencia de magmatismo de naturaleza alcalina vinculado temporoespacialmente con estructuras dilatantes sublatitudinales a NNO-SSE debidas al funcionamiento del SaLAM que afectan en extensión una corteza continental particularmente potente, ofrece interesantes objetivos prospectivos por rocas de tipo kimberlítico.

De este modo, se pueden realizar correlaciones tectónicas y geoeconómicas con otros territorios semejantes de los continentes africano y sudamericano mejor conocidos y ajustarse planes de muestreos diamantíferos en rocas primarias y sedimentos de corrientes aledaños a comarcas de la porción central del SaLAM.

Agradecimientos Se agradecen las correcciones indicadas por el Comité Editorial de la Revista Bresileira de Geociências. El presente trabajo es una contribución al Proyecto de Investigación UBACYT (TX 015).

\section{Referencias}

Bossi J., Ferrando L.A., Montaña J., Campal N., Morales H., Gancio F., Schipilov A., Piñeyro D. \& Sprechmann P., 1998. Carta Geológica del Uruguay, escala 1/500.000. Montevideo, Edición Geoeditores SRL, (CD-ROM).

Dalla Salda L., Bossi J. \& Cingolani C., 1988. The Rio de La Plata cratonic region Southwestern Gondwanaland. Episodes, 11:263-269.

Gómez-Rifas C.G. \& Masquelin-Arcelus H.C. 1996. Petrología y geoquímica de las rocas volcánicas cretácicas del Uruguay. In: AGA/Buenos Aires - Argentina, $\mathrm{XIII}^{\circ}$ Congreso Geológico Argentino y $\mathrm{III}^{\circ}$ Congreso de Exploración de Hidrocarburos, Actas, III: 635-652

Introcaso A. \& Huerta E., 1982. Interpretación del exceso de gravedad en Uruguay, In: Buenos Aires, Argentina/ $5^{\circ}$ Congreso Latinoamericano de Geología, Actas, IV: 87-104.

Muzio R., Peel E. \& Artur C., 1999. New geochronological data of the Valle Chico alkaline massif (Uruguay), using U-Pb and $\mathrm{Sm}-\mathrm{Nd}$ systematics. In: AGA/Córdoba - Argentina, II Simposio Sudamericano de Geología Isotópica, Extended abstracts (CD-rom).

Peate D.W., 1997. The Paraná-Etendeka Province. In: Mahoney J.J. \& Coffin M.F. (eds) Large igneous provinces, Continental: oceanic and planetary flood volcanism. American Geophysical Union, Geophysical Monograph
100:217-245.

Pirelli H., 1999. Contribución al conocimiento de la Formación Valle Chico, Uruguay. In: UNESP/SBG, Serra Negra - Brasil, $5^{\circ}$ Simpósio sobre o Cretáceo do Brasil y $1^{\circ}$ Simposio sobre el Cretácico de América del Sur, Anais, 433-437.

Rossello E.A., Santa Ana H. De, Veroslavsky G. 2001. El Lineamiento Santa Lucía-Aiguá-Merín (Uruguay): un corredor tectónico extensivo y transcurrente dextral precursor de la apertura Atlántica. Rev Bras Geoc, 30:749-756.

Servicio Geográfico Militar 1973. Carta Gravimétrica Provisoria de la República Oriental del Uruguay, a escala 1:1.000.000, Montevideo, Rep. Oriental Uruguay.

Turner S.P., Regelous M., Kelley S., Hawkesworth C.J. \& Mantovani M.S.M., 1994. Magmatism and continental break-up in the South Atlantic: High precision ${ }^{40} \mathrm{Ar}-{ }^{39} \mathrm{Ar}$ geochronology. Earth and Planetary Science Letters, 121:333-348.

Manuscrito NB - 038

Recebido em 25 de novembro de 200

Revisão do autores em 21 de dezembro de 2001 Revisão aceita em 22 de dezembro de 2001 